\title{
SPONTANEOUS RECOVERY OF FLUORESCENCE BY PHOTOBLEACHED SURFACE-ADSORBED PROTEINS
}

\author{
Andrea L. Stout ${ }^{1}$ and Daniel Axel.Rod*1,2 \\ 'Biophysics Research Division and 'Department of Physics, \\ University of Michigan, Ann Arbor, MI 48109, USA \\ (Received 9 November 1994; accepted 27 March 1995)
}

\begin{abstract}
Fluorescence photobleaching of a carboxyfluorescein-labeled protein (erythrocyte cytoskeletal protein 4.1) immobilized on bare glass is found to be spontaneously reversible, provided that the sample is deoxygenated. After a short (hundredths of seconds) photobleaching laser flash, the subsequent fluorescence excited by a dim probe beam partly recovers on a long (tenths of second) time scale, even in the absence of chemical exchange or diffusion processes. Neither the fraction of the fluorescence that bleaches reversibly nor its recovery rate is a strong function of fluorophore surface concentration. At a fixed surface concentration, the reversibly photobleached fraction and its recovery rate decreases with increasing duration or intensity of the bleaching flash. On the other hand, nondeoxygenated air-equilibrated samples exhibit almost total irreversible bleaching on this time scale. Quantitative fluorescence microscopy experiments occasionally require deoxygenation to avoid photochemical crosslinking or photobleaching or to enhance the triplet state population. The observations presented here indicate that fuorescence recovery after photobleaching (FRAP) experiments performed under deoxygenated conditions for measuring diffusion or chemical kinetics should be interpreted with caution: fluorescence recoveries may be due to intrinsic photochemical processes rather than fluorophore mobility. The recovery effect appears too slow to be ascribed simply to a relaxation of a triplet state; other possible explanations are offered.
\end{abstract}

\section{INTRODUCTION}

Fluorescence recovery after photobleaching $($ FRAP) $\dagger$ has become widely established as a method for measuring the rates of motion of membrane components, in particular translational diffusion,' rotational diffusion ${ }^{2}$ and adsorption/ desorption kinetics. ${ }^{3}$ The FRAP method relies on the phenomenon of photobleaching, in which a brief pulse of intense light is used to render fluorescent molecules in a welldefined area nonfluorescent. After the illumination is returned to a low intensity, the observed recovery of fluorescence due to exchange of bleached and unbleached fluorophores allows determination of kinetic parameters that characterize the dynamic process of interest. One of the assumptions made in the interpretation of FRAP data is that bleaching of fluorophores is irreversible. However, it has been shown ${ }^{2,4}$ that much bleaching is actually spontaneously reversible on a microsecond to millisecond time scale. We examine here bleaching that can be reversible even on a much longer (tenths of second) time scale.

The exact mechanisms by which any photobleaching takes place have yet to be elucidated. However, for irreversible bleaching, most evidence points to an irreversible oxidation of triplet state dye molecules by molecular oxygen in the singlet state. ${ }^{5-7}$ Under conditions where oxygen concentration is low, a pulse of high-intensity light may also induce

*To whom correspondence should be addressed.

tAbbreviations: CF, carboxyfluorescein; FITC, fluorescein isothiocyanate; FRAP, fluorescence recovery after photobleaching; TIR, total internal reflection. different photochemical reactions, such as the dimerization of dye molecules. ${ }^{8}$ In this paper we report a slow, spontaneous reversibility in the bleaching of substrate-attached fluorescein-labeled protein, observed only under deoxygenated conditions. Deoxygenation is often desirable in quantitative fluorescence microphotometry to avoid photoinduced crosslinking at surfaces, ${ }^{9}$ to avoid rapid photobleaching and to enhance the population of a triplet state. ${ }^{10}$

The phenomenon of slowly reversible photobleaching described here was first observed in the course of FRAP experiments intended to study the kinetics of binding of carboxyfluorescein (CF)-labeled protein 4.1 (a cytoskeletal protein) to the cytoskeletal-facing surface of erythrocyte membrane under total internal reflection (TIR) illumination." the experiments described here aimed at characterizing reversible bleaching, we choose a simpler system: CF-4.1 that remains irreversibly adsorbed to glass coverslips after extensive washing with CF-4.1-free bulk solution. Intrinsically reversible photobleaching appears to be the only reasonable interpretation for the effects reported here. The irreversibility of CF-4.1 adsorption in these samples ensures that any postbleach recovery of fluorescence is not due to binding/unbinding kinetics of labeled protein at the surface. Furthermore, the large bleaching area employed here also argues against a postbleach fluorescence recovery arising from lateral diffusion of CF-4.1 along the surface.

\section{MATERIALS AND METHODS}

Sample preparation. Glass coverslips (\#l thickness) were cleaned by boiling for $\mathrm{l}-2 \mathrm{~h}$ in a $20 \%$ solution of Linbro detergent (Flow Laboratories). They were rinsed repeatedly with tap water, then with 
distilled water and finally with $95 \%$ ethanol before being stored in a $110^{\circ} \mathrm{C}$ oven until use. Just prior to preparing samples for photobleaching experiments, cleaned coverslips were plasma cleaned under argon for $5 \mathrm{~min}$. (This treatment makes the surface of the glass hydrophilic, facilitating spreading and uniform coverage of the coverslips with an aqueous solution.) Then, $200 \mu \mathrm{L}$ of the desired CF4.1 protein solution was placed on each bare glass coverslip. These coverslips were incubated in the dark for $30-40 \mathrm{~min}$ at room temperature, after which time they were washed extensively with a buffer consisting of $100 \mathrm{~m} M \mathrm{KCl}, 20 \mathrm{mM} \mathrm{NaCl}, 25 \mathrm{~m} M$ Tris, pH 7.6, $0.2 \mathrm{~m} M$ EDTA and $0.2 \mathrm{~m} M$ dithiothreitol to wash away any reversible adsorption.

The sample coverslip was installed as the top coverslip in a Dvorak-Stotler perfusion charnber (Nicholson Precision Instruments, Inc.). The chamber was then filled and flushed three times with the above buffer before finally being filled with ejther air-saturated buffer or deoxygenated buffer. The chamber inlet and outlet ports were sealed for experiments done under conditions of low oxygen concentration. Deoxygenation was accomplished by bubbling nitrogen gas vigorously through the buffer before adding an oxygen-scavenging system consisting of glucose oxidase and catalase (Sigma) to final concentrations of 5 units $/ \mathrm{mL}$ and 150 units $/ \mathrm{mL}$, respectively. The excess of catalase is added to prevent the buildup of $\mathrm{H}_{2} \mathrm{O}_{2}$ produced during the oxidation of glucose.

Microscopv and data collection. Illumination of the sample either by standard EPI (through the objective lens) or TIR (via the evanescent field of total internal reflection) was employed in conjunction with a Leitz. Diavert inverted epifluorescence microscope with its dichroic mirror and barrier filter appropriate for fluorescein. I1lumination was provided by the $488 \mathrm{~nm}$ line of a $3 \mathrm{~W}$ argon-ion laser (Lexel, Palo Alto, CA). The intensity of the excitation light was controlled by an 80286-based computer (Zenith 248) ria an acousto-optic modulator (NEC Corporation, Mountain View, CA). The approximate photobleaching pulse intensity at the sample is reported along with each set of results. The intensity of the photobleaching pulse was $9000-10000$ times that of the probe illumination. For those experiments using TIR illumination, mirrors directed the beam to a cubical glass prism coupled to the top sample coverslip by immersion oil as in Burghardt and Axelrod. ${ }^{2}$ The beam, focused to a small spot by a lens located just upbeam from the prism, was incident on the sample-water interface at an angle of $\sim 74^{\circ}$. The resulting illumination spot was approximately $100 \mu \mathrm{m}$ long and 30 $\mu \mathrm{m}$ wide, with an intensity profile characterized by an elliptical Gaussian. Fluorescence from the sample was collected by a $40 x$. 0.75 numcrical aperture water-immersion objective (Zeiss), and an adjustable diaphragm at the image plane was used to control the size and location of the area on the sample from which fluorescence was collected. For those experiments using EPI illumination, the collimated incident laser beam was left unfocused as it entered the microscope's field diaphragm plane; the same $40 \times$ objective as above was used for fluorescence excitation and collection. This standard configuration resulted in a circular Gaussian illumination region at the sample with a characteristic radius of about $30 \mu \mathrm{m}$. For either the TIR or EPI illumination configurations, a photomultiplier tube (Hamamatsu R943-02) mounted atop the microscope's trinocular head and cooled to $-22^{\circ} \mathrm{C}$ by a Peltier housing (Pacific Instruments) with an internal amplifier-discriminator for photon counting detected fuorescence emitted from the sample.

Photon pulses from the amplifier-discriminator were sent to a counter/timer board (Keithley/Metrabyte CTM-05) installed in the 80286 computer. A custom program allowed the user to input $\mathrm{cx}$ perimental parameters such as sample times (generally selected at $25-100 \mathrm{~ms}$ ), duration of blcaching pulses (as presented in the figure captions) and duration of the post-bleach recording time. Generally, 3-10 repetitions of the bleach/recovery cycle were signal averaged to improve statistical signal/noise, with each repetition at a fresh spot on the sample. The counter/timer board also provided a transient pulse that electrically shorted (through an external transistor) the photomultiplier's photocathode and first dynode to protect the photomultiplier during the bright bleach flash.

Recovery curve fitting. In order to characterize the rate and extent of recovery, data were fitted to a single exponential function of the form:

$$
F(1)=F(-)-\left|a_{i}+a_{1} \exp (-k 1)\right|
$$

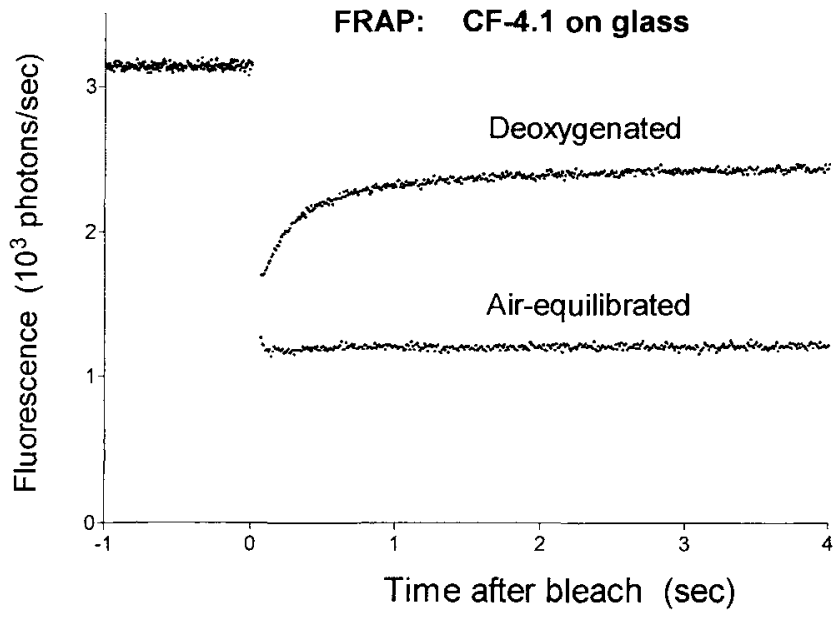

Figure 1. Actual FRAP (with EPI illumination) curves for CF-4.I immobilized on glass, as immersed in air-equilibrated buffer and deoxygenated buffer. All experimental parameters (CF-4. 1 concentration $=33 \mu \mathrm{g} / \mathrm{mL}$; laser intensity $=50 \mu \mathrm{W} / \mu \mathrm{m}^{2}$, bleach duration $=20 \mathrm{~ms}$, sample time $=10 \mathrm{~ms}$ ) are identical in the two cases. The air-equilibrated and deoxygenated results are averaged over 5 and 10 different bleach/recovery curves, respectively, each at fresh spots on the samples. The fluorescence units are kilo-photon counts pei sample time bin per single recovery. Note that the presence of oxygen makes the sample more bleachable.

where $\mathrm{F}(-)$ is the prebleach fluorescence. This fitting function was not derived from any particular model of the photochemistry of the recovery. Rather, it was chosen to provide a simple, quantitative estimate of the recovery rate and of the relative amounts of reversible and irreversible photobleaching. The fraction of irreversible bleaching is

$$
\mathrm{f}_{\mathrm{i}}=\mathrm{a}_{\mathrm{i}} /\left(\mathrm{a}_{\mathrm{i}}+\mathrm{a}_{\mathrm{i}}\right)
$$

\section{RESULTS}

\section{Oxygen dependence}

Figure I shows examples of TIR-FRAP data from samples consisting of CF-labeled protein 4.1 irreversibly immobilized on glass under (1) air-saturated buffer and (2) deoxygenated buffer. The behavior of the postbleach fuorescence is markedly different in the two cases. In the presence of oxygen the postbleach fluorescence is constant, as would be predicted for a case of irreversible bleaching where lateral motion and on/off kinetics do not take place. Under conditions where the oxygen concentration is very low, however, the postbleach fluorescence exhibits a recovery with a halftime typically around $0.5 \mathrm{~s}$. This recovery was also observed on samples of fluorescein isothiocyanate (FITC)-labeled anti-spectrin or CF-4.1 irreversibly adsorbed to the cytoplasmic surface of hemolyzed red cell membranes flattened on glass."

\section{Surface concentration dependence}

The absolute surface concentration of CF fluorophore that was irreversibly adsorbed to bare glass (through its covalent attachment to protein 4.1 ) could not be controlled directly over different sets of experiments, due to variations in dye/ protein ratio, local surface charge of the glass and freshness of the 4.1 preparation. However, within a particular set of experiments done with the same CF-4.1 preparation on the 

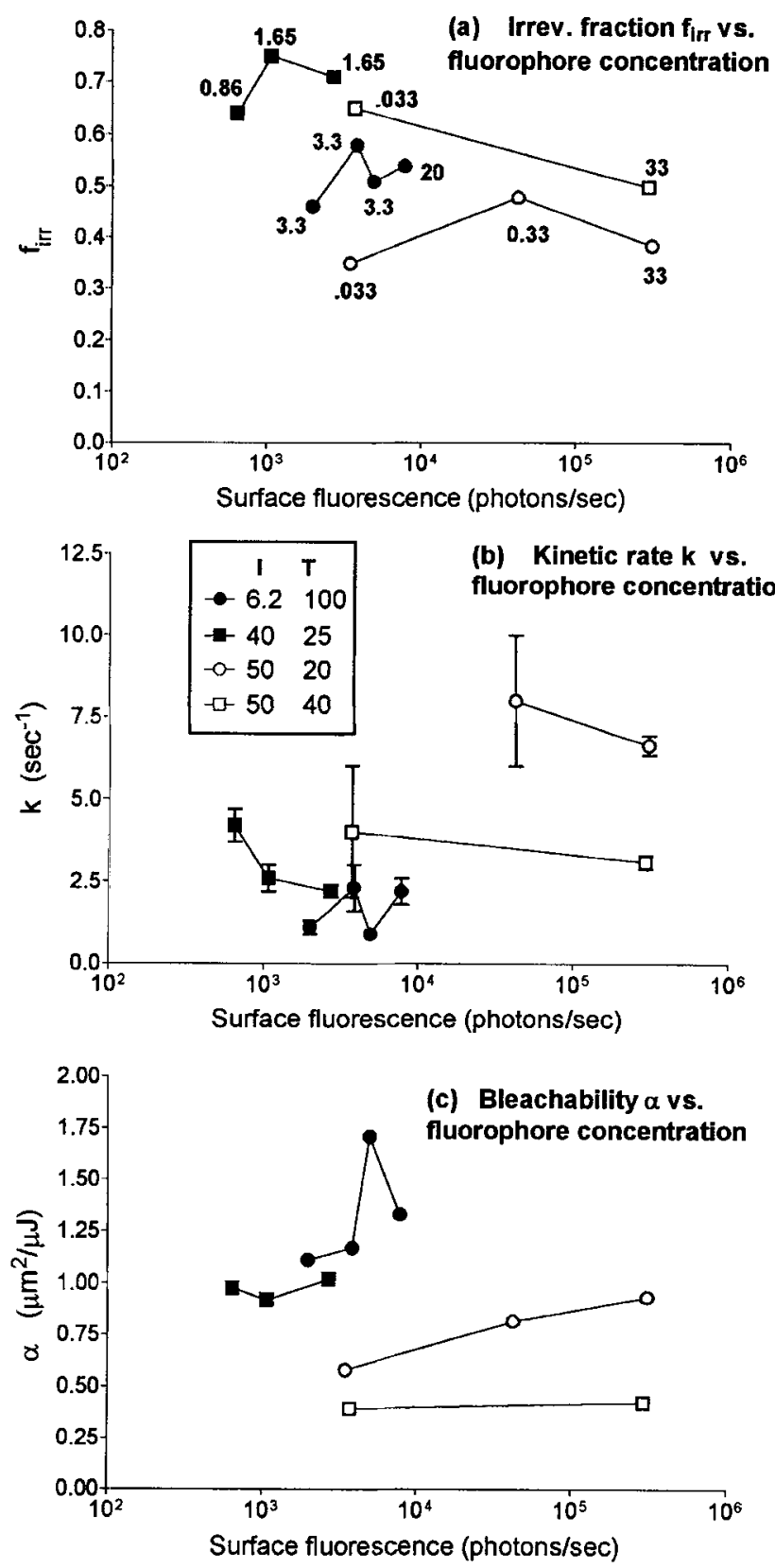

Figure 2. Surface fluorophore concentration dependence of the (a) irreversible fraction $\mathrm{f}_{\text {irr }}$, (b) recovery rate $k$ of the reversible component (as defined in Eq. 1 , in units of $\mathrm{s}^{-1}$ ) for CF-4.1 immobilized on glass and (c) total bleachability $\alpha$ (as defined by Eq. 3, in units of $\mu \mathrm{m}^{2} / \mu J$ ). Points from four different data sets, each with its own bleach intensities (approximate, in $\mu \mathrm{W} / \mu \mathrm{m}^{2}$ ) and durations (in $\mathrm{ms}$ ), are shown connected internally by thin solid lines. The data with closed symbols were based upon TIR optics and the open symbols upon EPI optics. The range of fluorophore relative surface concentrations was obtained as described in the text, in part from variability in binding to different regions of the surface, and in part from concentration changes in the mixture of bulk CF-4.1 and unlabeled $\mathrm{CF}$ 4.1 used to prepare each sample. The bulk CF-4.1 concentration (in $\mu \mathrm{g} / \mathrm{mL}$ ) actually used for each point is indicated by the nearby number in the top graph. The total protein concentration of the preparative bulk solution was always $33 \mu \mathrm{g} / \mathrm{mL}$ (except in one case with $20 \mu \mathrm{g} / \mathrm{mL}$ ) due to the addition of unlabeled 4.1 in some cases. The dye/protein molar ratio was approximately 2 for the points with closed symbols and about 1.3 for the open symbols.

The surface concentration was judged in a relative sense from the same set of glass slides and on the same day with the same optical alignment, relative surface concentrations could be measured as the fluorescence intensity detected from the surface. Within such sets, the relative surface concentration was varied in two ways: (1) In the bulk protein solution from which the surface coating was adsorbed, the ratio of fluorescent $\mathrm{CF}-4.1$ to unlabeled protein was adjusted, generally from pure CF-4.1 at $20-33 \mu \mathrm{g} / \mathrm{mL}$ to a $1: 1000$ dilution of the fluorescent species while keeping the total protein concentration (labeled + unlabeled) constant. (2) Even within a single sample, different locations on the surface displayed a considerable variation in fluorescence, presumably due to lateral nonuniformities in surface charge or roughness.

The size of the irreversible fraction $f_{i r r}$ seen on deoxygenated samples does not depend significantly on the surface concentration, as measured by the fluorescence intensity of the sample corrected to a constant illumination intensity (see Fig. 2a). Figure 2 a does show a considerable variation in $f_{\text {irr }}$ (ranging from around 0.3 to almost 0.8 ) over different sets of data with different preparations, bleaching intensities and bleaching durations. On a different kind of sample preparation-deoxygenated samples of CF-4.1 irreversibly bound to the cytoplasmic sides of glass-adhered red cell membranes" - $f_{\text {irr }}$ was as low as 0.2 . But within each data set in which all other conditions were held constant, changes in surface concentration over two orders of magnitude did not affect $\mathrm{f}_{\mathrm{irr}}$. It is still possible that variations of concentration well outside the range explored here might have an effect.

The kinetic rate $k$ of the fluorescence recovery was also not a significant function of surface concentration (Fig. 2b), although the error bars are much larger here than for $f_{\text {irr }}$.

It is also of interest to examine the total (reversible plus irreversible) bleachability of the samples as a function of surface concentration. We define total bleachability $\alpha$ in an oversimplified but useful manner, assuming that the postbleach intensity decreases as a simple exponential with the number of bleaching pulse photons (IT) to which the sample is exposed:

$$
\mathrm{F}(0)=\mathrm{F}(-) \mathrm{e}^{-\alpha \mathrm{IT}} .
$$

In actuality, the bleaching process may be multiexponential, representing different parallel or series processes and may even contain nonlinear terms in $I$ in the exponent $t^{4,13}$ or a concentration-dependent $\alpha .{ }^{13,14}$ But in the simplest single

$\leftarrow$

fluorescence (in photons/s) excited by a fixed intensity probe beam and detected through a fixed size image plane diaphragm in the microscope. This measurement is not calibrated and may not be exactly linear with actual fluorophore concentration (due to possible self-quenching at high concentrations) but it correctly depicts the trends within each data set. Because of the lack of calibration between sets, the position of each line-connected data set relative to the other sets along the abscissa is very approximate, and not meaningful at all in comparing EPI and TIRF data sets. The standard error uncertainties (originating from photon shot noise) as derived from the nonlinear regression fit to each recovery curve are shown; they do not appear only where the error is smaller than the point size on the graph. Each open symbol represents an average of 10 bleach/recovery runs. Each closed symbol represents an average over different numbers of runs, as follows in order of increasing surface fluorescence: closed circles, 15,7 and 4 runs; and closed squares, 50,20 and 15 runs. 
(a)

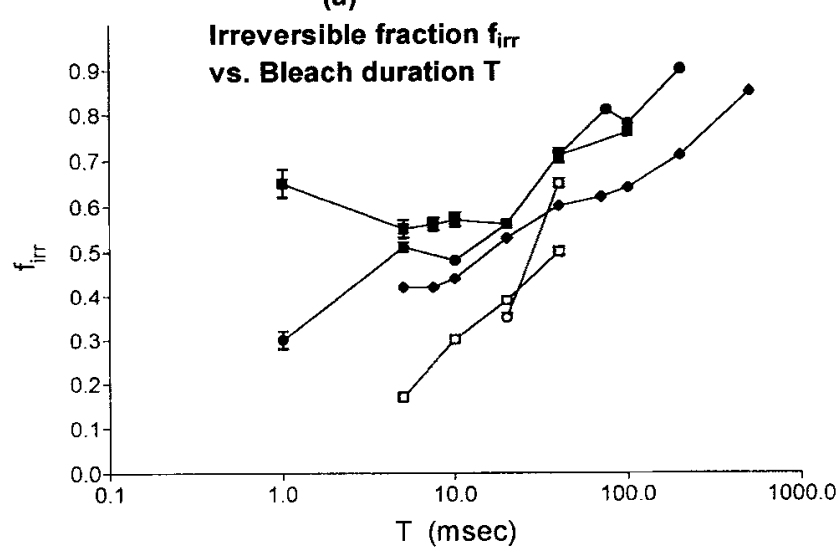

(b)

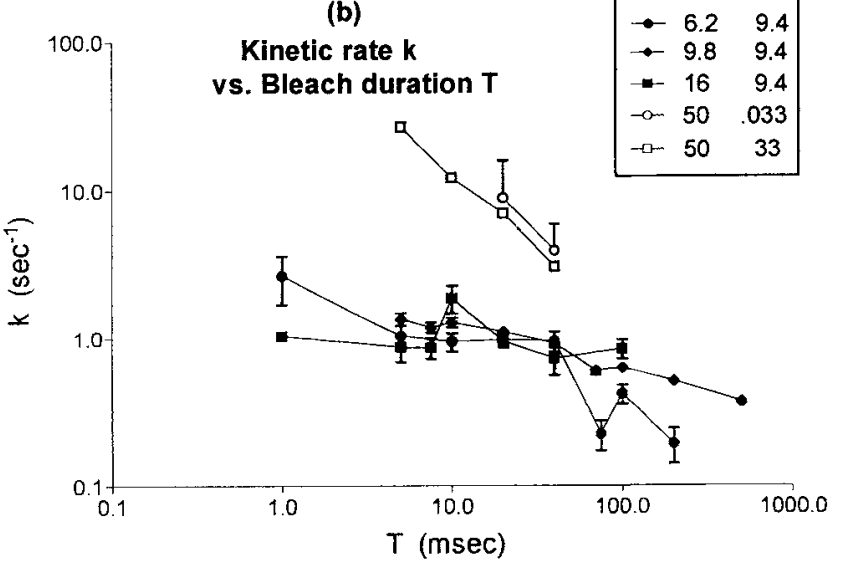

Figure 3. Bleach duration dependence on the (a) irreversible fraction $\mathrm{f}_{\mathrm{jir}}$ and (b) recovery rate $k$ for CF-4.1 immobilized on glass. For the different data sets, the bleach intensities (approximate, in $\mu \mathrm{W} / \mu \mathrm{m}^{2}$ ) and the CF- 4.1 bulk concentrations (in $\mu \mathrm{g} / \mathrm{mL}$ ) that were used in preparing the samples are indicated in the legend. The data with closed symbols were based upon TIR optics and the preparative bulk solution contained $260 \mu \mathrm{g} / \mathrm{mL}$ of unlabeled bovine serum albumin in addition to CF-4.1 (with a dye/protein molar ratio of 3 ). The data with open symbols were based upon EPI optics and for both relevant data sets, the preparative bulk solution contained a total (unlabeled plus CF-labeled at a dye/protein molar ratio of 1.3 ) of $33 \mu \mathrm{g} / \mathrm{mL}$ of protein 4.1. Each point represents an average of 5-10 bleach/recovery runs.

exponential model, bleachability $\alpha$ is proportional to the probability that a fluorophore becomes bleached per bleach pulse photon. Figure $2 \mathrm{c}$ plots bleachability $\alpha$ is surface concentration based on the same recovery curves from which the Fig. $2 a, b$ data were derived. It appears that higher surface concentrations are slightly more bleachable overall but the effect is not dramatic.

\section{Bleach duration dependence}

We examined the dependence of both the irreversible fraction and the reversible recovery rate upon bleach duration $\mathrm{T}$, for five different sets of bleach intensities and bulk concentrations and dilutions of CF-4.1 used in preparing the samples. Longer bleaching pulses lead to larger irreversibly bleached fractions (Fig. 3a) and slower reversible recovery rates (Fig. 3b).
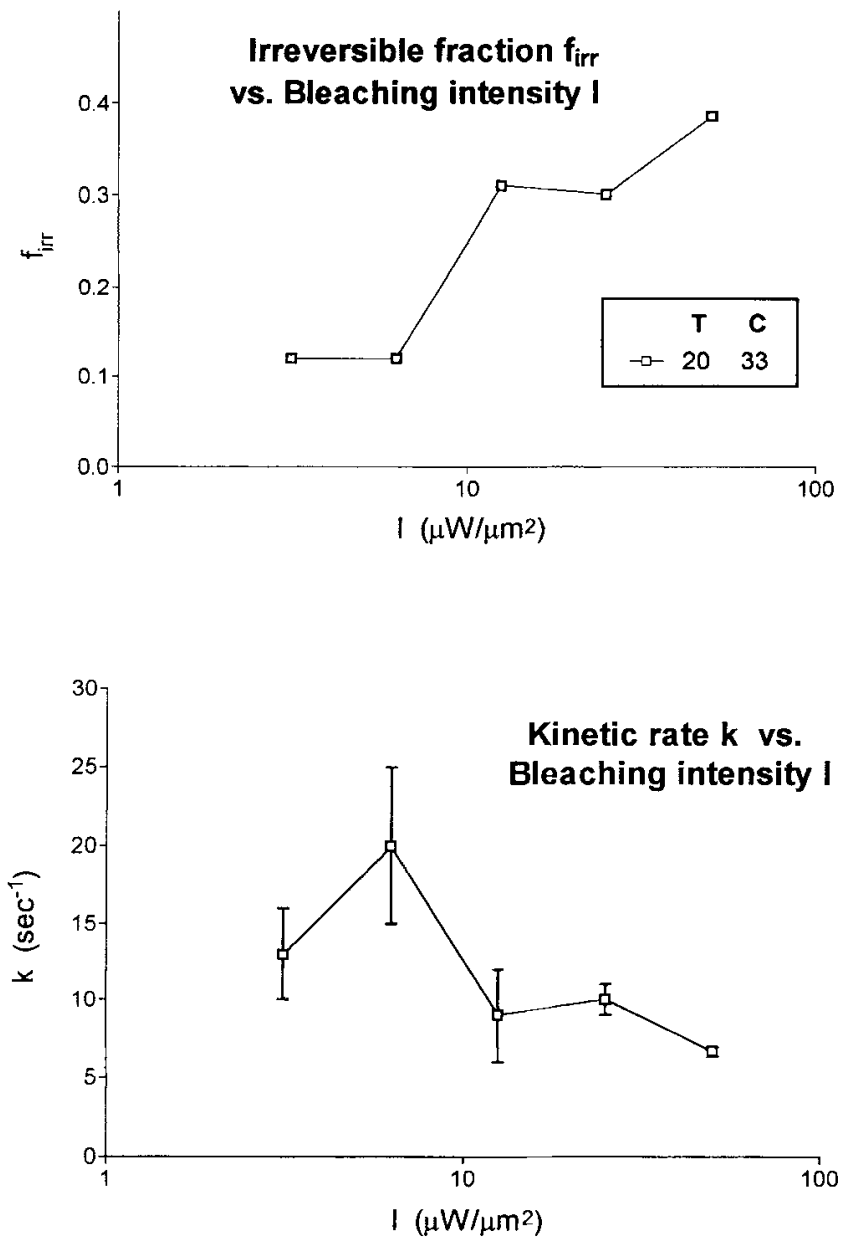

Figure 4. Bleach intensity dependence of the (a) irreversible fraction $\mathrm{f}_{\mathrm{irr}}$ and (b) recovery rate $k$ for CF-4.1 immobilized on glass. These data were based on EPI optics, the bleach duration was $20 \mathrm{~ms}$ and the preparative bulk solution contained $33 \mu \mathrm{g} / \mathrm{mL}$ of CF -4.1 (dye/ protein molar ratio of 1.3 ) with no unlabeled 4.1 . Each point represents an average of five bleach/recovery runs.

\section{Bleach intensity dependence}

For a single set of runs with a fixed bleached duration and CF-4. 1 concentration, increasing the intensity of the bleaching pulse I significantly increases the irreversibly bleached fraction (Fig. 4a). Increasing bleaching pulse intensity also tends to reduce the rate of recovery of the reversible fraction, although the uncertainties are large. In view of the similar effects of bleach duration, the key parameter may be the bleach photon number (IT).

\section{Size of bleached or observed area}

In principle, a fluorescent recovery could be due to diffusion of adsorbed protein along the surface. However, this possibility seems unlikely here. The characteristic size of the bleached region (at least $30 \mu \mathrm{m}$ for both TIR and EPI) was sufficiently large in all experiments such that only an unrealistically high surface diffusion coefficient of greater than about $5 \times 10^{-6} \mathrm{~cm}^{2} / \mathrm{s}$ could contribute to a fluorescent recovery on the $0.5 \mathrm{~s}$ time scale. Furthermore, varying the size of the illumination area or of the image plane diaphragm had 
no effect on either the irreversible fraction or the reversible recovery rate (data not shown). Therefore, the recovery of fuorescence was not due to lateral diffusion of labeled protein along the surface of the glass.

\section{DISCUSSION}

Reversible photobleaching has been observed in previous studies, both in deoxygenated and oxygenated systems..$^{2.10}$ Under the conditions used in those investigations, the time scale of the observed reversibility was in the microsecond to millisecond range, and the postbleach recovery could be ascribed to the relaxation of an excited triplet state. Here, the recovery is much longer, on the order of many tenths of seconds. This slowly reversible "bleaching" is observed only under deoxygenated conditions.

One speculation is that the phenomenon arises from an interaction between fluorophores on the surface. Under conditions where oxygen is scarce, flash-induced dimerization may be a more favorable reaction than photobleaching, for which a requirement for molecular oxygen has been well established. ${ }^{5.7}$ Reversible photodimerization of anthracene and tetracene under deoxygenated conditions has been reported, with the quantum yield for photodimer formation proportional to monomer concentration and the quantum yield for the reverse reaction a constant. ${ }^{8}$ Carboxyfluorescein has also been shown, along with other dyes, to form nonfluorescent dimers at high concentrations. ${ }^{15}$ It is possible that CF might also undergo a photoinduced dimerization under more dilute conditions as does anthracene. The lack of a clear dependence of the irreversibly bleached fraction upon surface fluorophore concentration (Fig. 2) would appear to argue against this hypothesis. On the other hand, the local concentration of fluorophore may be determined more by the dye/protein ratio and pattern of labeling sites on a single protein and the degree of microaggregation of CF-4.1 molecules on the surface than it is determined by the overall surface fluorophore concentration. The lack of a clear dependence of reversible recovery rate upon surface fluorophore concentration in these experiments also does not contradict the photodimerization hypothesis, because the reconversion of each dimer to monomers during the low intensity probe illumination would follow the same mechanism independently of its neighbors.

The increase in the irreversibly bleached fraction with increasing duration or intensity of the photobleaching pulse shown in Figs. 3a and 4a can be explained by the fact that the enzymatic deoxygenation method, while relatively thorough, does not remove $100 \%$ of the molecular oxygen from solution. Consider two competing pathways that a particular dye molecule could follow during a bleaching flash:

Irreversible: $\quad \mathrm{G} \stackrel{\mathrm{hv}}{\rightarrow} \mathrm{S} \rightarrow \mathrm{T} \stackrel{\mathrm{O}_{2}}{\rightarrow}$ (monomer $^{*}{ }^{*}$ irr

$$
\text { Reversible: } \quad \mathrm{G} \stackrel{\text { ho }}{\rightarrow} \mathrm{S} \rightarrow \mathrm{T} \stackrel{\mathrm{G}}{\rightarrow}\left(\text { dimer) }{ }^{*} \text { rev } \stackrel{\text { slow }}{\rightarrow} \mathrm{G}+\mathrm{G}\right. \text {. }
$$

$G, S$ and $T$ denote the monomeric dye molecule in the ground, excited singlet and triplet states, respectively. An asterisk represents a nonfluorescent species. The first pathway leads to the dead-end (irreversible) production of bleached fluorophores and is most likely in the presence of oxygen. In cases with only very few oxygen molecules near a particular monomer, the second of the two pathways, with its slow reversion to the $G$ state, is more likely to occur during a brief bleaching flash, although a few triplet state monomers still would be near enough to an oxygen molecule to get irreversibly bleached. However, during longer flashes (longer than the characteristic reversible recovery time, generally around $0.1 \mathrm{~s}$ or longer) fluorophores could undergo the monomer-dimer cycle a few times, and during each pass through the cycle some triplet monomers would find an oxygen molecule, thereby increasing the yield of the irreversible bleaching process. Likewise, a brighter flash increases the probability that a fluorophore traversing the second (reversible bleaching) pathway will become excited again during the pulse duration, thereby increasing the overall probability of eventual irreversible bleaching. However, for bleach durations much shorter than the characteristic lifetime of the putative dimer, the effect of brighter bleaching should be rather small.

The moderate but significant decrease in recovery rate with increasing bleach duration and possibly with intensity may be more complicated. This effect does not appear to result from any local temperature increase induced by the bleaching flash. For a bleaching pulse incident power of 0.12 $W$, a length of a shorter axis of the ellipsoidal spot of $\sim 30$ $\mu \mathrm{m}$ and a thermal diffusivity of $1.4 \times 10^{-3} \mathrm{~cm}^{2} \mathrm{~s}^{-1}$ for water (neglecting the difference between thermal properties of water and glass), the approximate steady-state temperature increase that would be reached after a very long bleaching pulse turns out to be only $0.018^{\circ} \mathrm{C}^{16}$ Apart from heating, a possible explanation for the effect of bleach duration on recovery rate is that during extended periods of high-intensity illumination, a multiplicity of dye-dye interactions begin taking place. Short flashes of light may induce a single species of dimer formation, which has a characteristic relaxation rate around $2 \mathrm{~s}^{-1}$ or so. During longer flashes, more stable dimers with longer relaxation times may form, or perhaps even multimers of three or more dye molecules. The possible complexity of the process is also suggested by the significant variation of recovery rates seen on different samples, even with the same bleach duration. Additional experiments examining the spectral characteristics of the postbleach dye population formed with and without oxygen could provide more insight into the exact nature of the processes taking place under these conditions.

Slowly reversible photobleaching of CF-labeled cytoplasmic protein was also observed on another kind of surface: human red cell membranes adhered and hemolyzed at glass coverslips (see Fulbright and Axelrod 9 for preparation details). These glass-supported flattened erythrocyte membranes, which display their cytofacial surfaces to the solution, were exposed to either CF-4.1 or to FITC-labeled antibodies to spectrin (another component of the red cell membrane skeleton) and then washed extensively with fluorophore-free buffer as above to remove reversible adsorption. Observations were made on these biological samples in order to eliminate the possibility that the reversible photobleaching effects were somehow limited to fluorescent proteins directly adsorbed to bare glass.

The existence of a long duration (tenths of a second or longer) postbleach recovery on deoxygenated samples that 
has nothing to do with the usual parameters measured in FRAP experiments (translational and rotational diffusion coefficients and adsorption kinetic rates) means that FRAP results performed under deoxygenated conditions should be interpreted with caution.

Acknowledgements-The authors thank Dr. Watt W. Webb for useful discussions about the possible origin of these effects. This work was supported by NIH NS 14565, NSF DMB 8805296, and NSF MCB 9405928 to D.A.

\section{REFERENCES}

1. Axelrod, D., D. E. Koppel, J. Schlessinger, E. L. Elson and W. W. Webb (1976) Mobility measurements by analysis of fluorescence photobleaching recovery kinetics. Biophys. J. 16. 1055-1069

2. Velez, M. and D. Axelrod (1988) Polarized fluorescence photobleaching recovery for measuring rotational diffusion in solutions and membranes. Biophys. J. 53, 575-591.

3. Thompson, N. L., T. P. Burghardt and D. Axelrod (1981) Measuring surface dynamics of biomolecules by total internal reflection with photobleaching recovery or correlation spectroscopy. Biophys. J. 33, 435-454.

4. Yuan, Y. and D. Axelrod (1994) Photobleaching with a subnanosecond laser flash. J. Fluor. 4, 141-151.

5. Foote, C. S. (1968) Mechanisms of photosensitized oxidation Science 162, 963-970.

6. Sheetz, M. P. and D. E. Koppel (1979) Membrane damage caused by irradiation of fluorescent concanavalin A. Proc. Natl. Acad. Sci. USA 76, 3314-3317.

7. Singh, R. J., J. B. Feix and B. Kalyanaraman (1992) Photobleaching of merocyanine 540: involvement of singlet molecular oxygen. Photochem. Photobiol. 55, 483-489.

8. Wei, K. S. and R. Livingston (1967) Reversible photodimerization of anthracene and tetracene. Photochem. Photobiol. 6, $229-232$.

9. Fulbright, R. M. and D. Axelrod (1993) Dynamics of nonspecific adsorption of insulin to erythrocyte membranes. J. Fluor. 3, $1-16$.

10. Yoshida, T. M. and B. G. Barisas (1986) Protein rotational motion in solution measured by polarized fluorescence depletion. Biophys. J. 50, 41-53.

11. Stout, A. L. and D. Axelrod (1994) Reversible binding kinetics of a cytoskeletal protein at the erythrocyte submembrane. Biophys. J. 67, 1324-1334.

12. Burghardt, T.P. and D. Axelrod (1981) Total internal reflection/ fluorescence photobleaching recovery study of serum albumin adsorption dynamics. Biophys. J. 33, 455-468.

13. Nakhimovsky, L., D. Chester, R. Hechtman and D. E. Koppel (1990) Photobleaching kinetics of NBD-PE and DiIC18 in DOPC multibilayer membranes. Biophys. J. 57, 481 a.

14. Bjarneson, D. W. and N. O. Petersen (1991) Effects of second order photobleaching on recovered diffusion parameters from fluorescence photobleaching recovery. Biophys. J. 60, 1128 1131.

15. Chen, R. F. and J. R. Knutson (1988) Mechanism of fluorescence concentration quenching of carboxyfluorescein in liposomes: energy transfer to nonfluorescent dimers. Anal. Biochem. 172, 61-77

16. Axelrod, D. (1977) Cell surface heating during fluorescence photobleaching recovery experiments. Biophys. J. 18, 129-131. 